\title{
Innovation and Crowdsourcing Contests
}

\author{
Laurence Ales · Soo-Haeng Cho · Ersin Körpeoğlu
}

\begin{abstract}
In an innovation contest, an organizer seeks solutions to an innovation-related problem from a group of independent agents. Agents, who can be heterogeneous in their ability levels, exert efforts to improve their solutions, and their solution qualities are uncertain due to the innovation and evaluation processes. In this chapter, we present a general model framework that captures main features of a contest, and encompasses several existing models in the literature. Using this framework, we analyze two important decisions of the organizer: a set of awards that will be distributed to agents and whether to restrict entry to a contest or to run an open contest. We provide a taxonomy of contest literature, and discuss past and current research on innovation contests as well as a set of exciting future research directions.
\end{abstract}

\section{Introduction}

"Everybody has a creative potential and from the moment you can express this creative potential, you can start changing the world." - Paulo Coelho

“Our best ideas come from clerks and stockboys." — Sam Walton

Many organizations today look beyond their boundaries to elicit innovation. With advances in information technology and global access to skilled individuals, contests (also known as tournaments) have emerged as a popular and cost-effective tool to elicit innovative solutions to challenging problems. A contest usually starts when a contest organizer announces a problem along with contest rules such as a set of awards (called "award scheme") and whether the contest is open to the public or not. Then, agents who are interested in the contest make efforts to develop solutions to the problem, and submit them to the organizer. Finally, the organizer evaluates these solutions, and awards the best one(s) according to the announced rule.

Laurence Ales

Tepper School of Business, Carnegie Mellon University, Pittsburgh, PA 15213, e-mail: a les@cmu . edu

Soo-Haeng Cho

Tepper School of Business, Carnegie Mellon University, Pittsburgh, PA 15213, e-mail: soohaeng@andrew . cmu . edu

Ersin Körpeoğlu

School of Management, University College London, London, UK E14 5AA, e-mail: e. korpeoglu@ucl.ac.uk 
This chapter focuses on two popular types of contests: innovation and crowdsourcing contests. In an innovation contest, an organizer seeks solutions to an innovation-related problem from a (not necessarily large) group of agents, and in a crowsourcing contest, the organizer seeks (not necessarily innovative) solutions from a large group of agents. While pointing out this subtlety, we will refer to both types of contests as "innovation contests" throughout the chapter as these contests mostly overlap in practice. To illustrate how these contests work in practice, consider the following example from Ales et al. (2016) (see their introduction for detailed examples). Since 2012, Samsung has organized several innovation contests, called Samsung Smart App Challenge, seeking innovative apps for its products. The contest started with Samsung's announcement of contest rules. For example, Samsung Smart App Challenge 2013 for GALAXY S4 was open to anyone who wished to participate, and distributed a total of $\$ 800,000$ prizes for top ten apps. The judging criteria were uniqueness, commercial potential, functionality, usability, and design.

Innovation contests are utilized for a broad set of topics ranging from mining solutions (e.g., Goldcorp Challenge which seeks proposals for identifying potential gold mining targets) to design (e.g., a logo design contest for FIFA World Cup) and software development (e.g., Samsung Smart App Challenge). While some organizations run their own contests, others employ contest platforms such as Challenge.gov, Ennomotive, InnoCentive, Inocrowd, and TopCoder that intermediate contests on behalf of their clients. For example, InnoCentive crowdsources innovation on behalf of a diverse group of clients such as AARP Foundation, Eli Lilly, NASA, and P\&G (InnoCentive 2017). InnoCentive organizes ideation, theoretical, and reductionto-practice (RTP) challenges (in which agents develop ideas, theoretical solutions, and prototypes, respectively) in subject areas such as chemistry and social innovation. Agents of different background compete in these free-entry open innovation contests for awards ranging from $\$ 5,000$ to $\$ 1$ million. As another example, TopCoder crowdsources software solutions on behalf of a large client base including Best Buy, Comcast, HP, and IBM (TopCoder 2017). Agents around the world compete in various software development contests that are open to public, and winners are awarded cash prizes around $\$ 10,000$, and the performance of all participants is converted into a continually updated TopCoder rating.

Through open innovation processes, an organizer can tap into a large number of experts outside of its firm boundary, and can select the most promising solution from many submitted solutions. Despite this benefit of having a large number of participants, the organizer does not need to pay every participant, the organizer may pay only one agent, the "winner,"who has submitted the best solution. In such a winner-take-all contest, all agents except the winner bear all the costs of their efforts. Yet, with many contest participants, agents expect their individual chance of winning a contest to be low, and hence may not have sufficient incentives to exert their best efforts. Thus, the contest organizer should carefully choose the right award scheme, and determine whether to restrict the number of participants to increase the probability of winning for individual agents. 
The objective of this chapter is to present a general model framework for innovation contests, and provide insights into two of the organizer's decisions that are essential from both practical and theoretical points of view. The first decision we study is a set of awards through which an organizer incentivizes agents to participate in a contest and make costly efforts. From a practical point of view, it is important for an organizer to assess when to adopt a winner-take-all award scheme and when to offer multiple prizes. From a theoretical point of view, the winner-take-all award scheme is almost a standard assumption in the contest literature, and it is important to determine when this assumption is justified. The second decision we analyze in this chapter is whether it is optimal for a contest organizer to hold an open contest without restricting entries to the contest. From a practical point of view, this analysis addresses when open innovation initiatives that rely on the "wisdom of crowds" are desirable. From a theoretical point of view, several papers in the contest literature implicitly assume that an organizer does not impose any entry restrictions to agents. Thus, it would be interesting to examine when it is indeed optimal for an organizer to hold an open contest. Because this decision of an organizer is closely related to agents' incentives, we will also discuss how participating agents change their level of effort when additional agents enter the contest.

While discussing award scheme and entry restriction decisions based on Ales et al. $(2016,2017)$ and Körpeoğlu and Cho (2017), we present a general model framework that encompasses several models that have been studied in the literature. In particular, our framework captures the following three main features of typical innovation contests in practice:

- When an organizer seeks the best $\mathrm{K}$ solutions, where $\mathrm{K}$ is a positive integer between one and the total number of participants, Ales et al. (2016) say that there are K "contributors" among participants in a contest. In some contests, an organizer is interested in only the best solution, so K equals one (cf. Taylor 1995). For example, in a logo design contest for FIFA World Cup, the organizer was interested in finding the best logo to adopt. In other contests, the organizer seeks several good solutions; for example, Samsung sought many useful apps in Smart App Challenge.

- Following Ales et al. (2017), we consider two sources of uncertainty that agents face. The first source of uncertainty is referred to as "technical uncertainty," and this stochastic element is often modeled as a search process for the best solution from a number of trials (e.g., Dahan and Mendelson 2001). For example, a logo designer may experiment on several logo sketches, and s/he does not know the results of those experiments a priori. The second source of uncertainty, called "taste uncertainty," is due to the subjective or unknown taste of the organizer. For example, in Samsung Smart App Challenge, when submitting their apps, developers do not know how judges will evaluate their apps in subjective criteria such as uniqueness, usability, and design.

- We model agents' heterogeneity utilizing a "productivity-based" model introduced by Körpeoğlu and Cho (2017). In this model, agents are heterogeneous in their productivity levels so that one unit of effort from a high-productivity agent creates higher value than that from a low-productivity agent. In practice, 
agents can feature heterogeneous productivity levels due to difference in experience, expertise, and overall ability. For instance, the TopCoder rating of an agent can indicate his/her ability or experience level because high rating indicates successful past performance.

The remainder of this chapter is organized as follows. In $\S 2$, we present our general model framework and discuss how this model framework encompasses existing models in the literature. In $\S 3$, we provide a brief taxonomy of the literature, and discuss several interesting work in the area of innovation contests. Then, we study the organizer's the award scheme and entry restriction decisions in $\S 4$ and $\S 5$. While we choose to focus on the above two decisions, we discuss some exciting open questions in $\S 6$.

\section{A General Model Framework for Innovation Contests}

In this section, we describe a fairly general environment that encompasses commonly used models when studying innovation contests. As discussed in $\S 1$, this general model essentially combines the models used in Ales et al. $(2016,2017)$ and Körpeoğlu and Cho (2017). In what follows, we first present our model of agents, and then we present our model of the organizer. At the end of this section, we briefly discuss this model in comparison to other models in the literature.

Agents Suppose that there are $N(\geq 2)$ agents who can participate in the contest. Each participating agent $i(\in\{1,2, \ldots, N\})$ develops a solution to the problem posed in the contest with solution quality (hereinafter "output") $y_{i} \in \mathcal{Y} \subseteq \mathbb{R} \cup\{-\infty, \infty\}$. Following Ales et al. (2017), we represent agent $i$ 's output as a function of improvement effort $q_{i}$, a number of trials $m_{i}$, trial shocks $\left(\widetilde{\epsilon}_{i 1}, \ldots, \widetilde{\epsilon}_{i m_{i}}\right)$, and a taste shock $\widetilde{\varepsilon}_{i}$ as follows:

$$
y\left(q_{i}, m_{i}, \widetilde{\epsilon}_{i 1}, \ldots, \widetilde{\epsilon}_{i m_{i}}, \widetilde{\varepsilon}_{i}\right)=v\left(q_{i}\right)+\max \left\{\widetilde{\epsilon}_{i t}, t=1, \ldots, m_{i}\right\}+\widetilde{\varepsilon}_{i}
$$

This function combines the following three components. First, each agent $i$ can exert "improvement effort" $q_{i}$, and this effort leads to a deterministic improvement $v\left(q_{i}\right)$ of agent's output, where $v$ is an increasing and concave function of $q_{i}$. Second, each agent $i$ may engage in a trial-and-error process by conducting several experiments, where the agent determines a number of trials $m_{i}$ (hereinafter, "trial effort"). In each trial $t\left(=1,2, \ldots, m_{i}\right)$, the agent faces uncertainty in the outcome of a trial, which is modeled through a trial shock $\widetilde{\epsilon}_{i t}$ that follows a Gumbel distribution with $E\left[\widetilde{\epsilon}_{i t}\right]=0$ and scale parameter $\mu$. (Throughout the chapter, we use the notation " $~$ " to represent random variables.) Each agent observes the outcome of these trials $\left(\widetilde{\epsilon}_{i 1}, \ldots, \widetilde{\epsilon}_{i m_{i}}\right)$ and submits the best one to the organizer. Third, each agent $i$ 's output is subject to the taste of the organizer, which we model by a taste shock $\widetilde{\varepsilon}_{i}$. The taste shocks of agents, $\widetilde{\varepsilon}_{i}$ 's, are independent and identically distributed (i.i.d.) random variables with a general disribution and $E\left[\widetilde{\varepsilon}_{i}\right]=0$. Unlike trial shocks $\widetilde{\epsilon}_{i t}$ 's, each agent $i$ is uncertain about the taste shock $\widetilde{\varepsilon}_{i}$ even after the development process is over. For practical examples and details of these components, see Ales et al. (2017).

We next define a general form for the utility of agent $i, U_{a}\left(q_{i}, m_{i}, x_{i}, c_{i}\right): \mathbb{R}_{+}^{4} \rightarrow \mathbb{R}$, which is defined over improvement effort $q_{i}$, trial effort $m_{i}$, monetary compensation $x_{i}$ received from the organizer, and 
heterogenous cost coefficient $c_{i}$ for exerting effort. The parameter $c_{i}$ is a privately known cost coefficient for agent $i$, drawn from a continuous distribution $\Phi$ similar to Moldovanu and Sela (2001). The utility of the agent takes the following form:

$$
U_{a}\left(q_{i}, m_{i}, x_{i}\right)=x_{i}-\psi\left(c_{i}\left(\tau_{1} q_{i}+\tau_{2} m_{i}\right)\right)
$$

where $\tau_{1}>0, \tau_{2}>0$, and $\psi$ is convex and increasing with $\psi(0)=0$. This utility function is more general than Ales et al. $(2016,2017)$ which consider identical agents, but it is similar to Körpeoğlu and Cho (2017). We define total effort as $e_{i}=\tau_{1} q_{i}+\tau_{2} m_{i}$. For example, for an agent with improvement effort $q_{i}$ and trial effort $m_{i}$, total effort $e_{i}$ may represent the total labor hours an agent spends, where $\tau_{1}$ and $\tau_{2}$ are time required for one unit of improvement and trial effort, respectively. Agent $i$ 's cost of making effort $e_{i}$ is $\psi\left(e_{i}\right)=\psi\left(c_{i}\left(\tau_{1} q_{i}+\tau_{2} m_{i}\right)\right)$.

The following lemma shows that the output function $y$ given in (1) can be simplified to a new function that depends only on the agent's total effort $e_{i}$ and aggregate shock $\widetilde{\xi}_{i}$. The first part of the lemma is shown by Ales et al. (2017) and a special case of the second part of the lemma is shown by Körpeoğlu and Cho (2017) under linear cost of effort and no output uncertainty. We present the proof of Lemma 1(b) in Appendix.

Lemma 1. (a) (Lemma 1 of Ales et al. 2017) The output function in (1) can be simplified to $y\left(e_{i}, \widetilde{\zeta}_{i}\right)=r\left(e_{i}\right)+\widetilde{\xi}_{i}$ in which $e_{i}$ is the total effort, $r$ is a concave and increasing function, and $\widetilde{\xi}_{i}$ is a random shock that is independent of $e_{i}$. For example, if $v\left(q_{i}\right)=\kappa \log \left(q_{i}\right)$ for some $\kappa>0$, then $r\left(e_{i}\right)=\gamma+\theta \log \left(e_{i}\right)$ where $\theta(>0)$ and $\gamma$ are constants.

(b) (Adapted from Körpeoğlu and Cho 2017) The cost-based model in which agents are heterogeneous in their cost coefficients and the output function $y\left(e_{i}, \widetilde{\xi}_{i}\right)=r\left(e_{i}\right)+\widetilde{\xi}_{i}$ is equivalent to a productivity-based model with $y\left(a_{i}, e_{i}, \widetilde{\xi}_{i}\right)=$ $r\left(a_{i} e_{i}\right)+\widetilde{\xi}_{i}$, where $a_{i}$ is agent $i$ 's heterogeneous productivity level drawn from distribution $G\left(a_{i}\right)=1-\Phi\left(1 / a_{i}\right)$ with support $[\underline{a}, \bar{a}]$, and the cost of effort is $\psi\left(e_{i}\right)$.

In the rest of this chapter, we use the simplified output function $y\left(a_{i}, e_{i}, \widetilde{\zeta}_{i}\right)=r\left(a_{i} e_{i}\right)+\widetilde{\zeta}_{i}$, and refer to $a_{i}$ as agent $i$ 's productivity, $e_{i}$ as agent $i^{\prime}$ s effort, and $\widetilde{\xi}_{i}$ as agent $i$ 's output shock. This model adds uncertainty to the productivity-based model introduced by Körpeoğlu and Cho (2017), and adds heterogeneous productivity levels to the model of Ales et al. (2016). The productivity level $a_{i}$ is drawn from a general distribution $G$ over the support $[\underline{a}, \bar{a}]$. Let $\widetilde{a}_{(j)}^{N}, G_{(j)}^{N}$, and $g_{(j)}^{N}$ represent the random variable, the distribution function, and the density function of the $j$-th highest productivity level among $N$ agents, respectively. It is not difficult to verify that $g_{(j)}^{N}\left(a_{i}\right)=\frac{N !}{(j-1) !(N-j) !}\left(1-G\left(a_{i}\right)\right)^{j-1} G\left(a_{i}\right)^{N-j} g\left(a_{i}\right)$. The output shock $\widetilde{\zeta}_{i}(\in \Xi)$ follows cumulative distribution $H$ and density $h$ with $E\left[\widetilde{\xi}_{i}\right]=0$ and $\Xi=[\underline{s}, \bar{s}]$ where $\underline{s} \in \mathbb{R} \cup\{-\infty\}$ and $\bar{s} \in \mathbb{R} \cup\{\infty\}$. Similarly, let $\widetilde{\xi}_{(j)}^{N}, H_{(j)}^{N}$, and $h_{(j)}^{N}$ represent the random variable, the distribution function, and the density function for the $j$-th highest value among $N$ output shocks, respectively. 
The Organizer The profit of the organizer, $\widehat{\Pi}(Y, X): \mathcal{Y}^{N} \times \mathbb{R}^{N} \rightarrow \mathbb{R}$, is defined over the output vector $Y$ and the compensation vector $X$. Following Ales et al. (2016), we consider the case where the organizer benefits from the best $K \in\{1, \ldots, N\}$ outputs, and refer to those agents who produce the best $K$ outputs as "contributors." Formally, we can extend the definition of a contributor in Ales et al. (2016) (who assume that only the best output is awarded a fixed prize) by utilizing a general compensation vector as follows:

Definition 1. Let $Y^{(K)}=\left\{y_{(1)}[Y], \ldots, y_{(K)}[Y]\right\}$ where $y_{(j)}[Y]$ represents the $j$-th highest output in $Y$ - for ease of notation, we use $y_{(j)}$ in short. The organizer's profit has $K$ contributors if for all $Y \in \mathcal{Y}^{N}, X \in \mathbb{R}_{+}^{N}$,

(i) There exists a continuously differentiable function $\Pi$ so that $\widehat{\Pi}(Y, X)=\Pi\left(Y^{(K)}, X\right)$;

(ii) For all $j=1,2, \ldots, K, \frac{\partial \Pi\left(Y^{(K)}, X\right)}{\partial y_{(j)}}>0$.

A compensation rule $\phi: \mathcal{Y}^{N} \rightarrow \mathbb{R}^{N}$ maps the output vector $Y=\left(y_{1}, \ldots, y_{N}\right)$ to a vector of compensations the organizer pays to agents, $X=\left(x_{1}, \ldots, x_{N}\right)$. As in many contests in practice, we restrict attention to the relative (also called ranked-order) compensation rule which compensates each agent based on the agent's relative rank of the output. Formally, a compensation rule is called the relative compensation rule when there exists some constant $A_{(j)}$ such that $\phi_{i}\left(y_{(j)}[Y]\right)=A_{(j)}$ for all $i \in \mathcal{N}, j=\{1, \ldots, N\}$ and $Y \in \mathcal{Y}^{N}$. Thus, the relative compensation rule consists of a vector of $N$ prizes (awards), denoted by $\left(A_{(1)}, \ldots, A_{(N)}\right)$, such that the agent who produces the $j$-th best output receives a prize of $A_{(j)}$. We refer to this vector of prizes as "award scheme." Furthermore, we refer to the agent who produces the best output as the winner, and to the award scheme that awards only the winner as the winner-take-all (hereinafter WTA) award scheme.

With $K$ contributors, the organizer's profit function under the relative compensation rule is:

$$
\Pi\left(Y^{(K)},\left(A_{(1)}, A_{(2)}, \ldots, A_{(N)}\right)\right)=\sum_{j=1}^{K} y_{(j)}-\sum_{j=1}^{N} A_{(j)} \quad \forall Y \in \mathcal{Y} .
$$

Whereas Ales et al. (2016) consider a general utility function for the organizer that can allow risk aversion and other complex functional forms (see Section 5 in Ales et al. 2016), in this chapter, we restrict attention to a risk-neutral organizer who maximizes profit as in Ales et al. (2017) and Körpeoğlu and Cho (2017).

We say that the organizer holds an "open contest" when all agents who wish to participate in a contest are allowed to do so. An open contest proceeds in the following sequence. First, the organizer announces the award scheme $\left(A_{(1)}, A_{(2)}, \ldots, A_{(N)}\right)$. Then, each agent $i \in\{1,2, \ldots, N\}$ privately learns a productivity level $a_{i}$, and then determines whether to participate in the contest and chooses an effort level $e_{i}$. An agent who chooses not to participate receives reservation utility 0 . Each agent $i$ who chooses to participate in the contest incurs the cost of effort $\psi\left(e_{i}\right)$. Next, each agent observes an output shock $\widetilde{\xi}_{i}$, and produces an output $y_{i}=r\left(a_{i} e_{i}\right)+\widetilde{\zeta}_{i}$. Finally, the contest organizer collects solutions of all participating agents, and gives awards to agents based on the award scheme $\left(A_{(1)}, A_{(2)}, \ldots, A_{(N)}\right)$. We assume that all parameters except the productivity level $a_{i}$ are common knowledge to both agents and the organizer, and we focus on symmetric pure-strategy Nash equilibria in which all agents with the same productivity level make the same effort. 
We next present the agent's and organizer's problems. Because Ales et al. $(2016,2017)$ and Körpeoğlu and Cho (2017) consider either agent uncertainty or heterogeneity, we will develop an original formulation that encompasses the formulations in those papers. Let $e^{*}:[\underline{a}, \bar{a}] \rightarrow \mathbb{R}_{+}$denote the equilibrium effort function, where $e^{*}\left(a_{i}\right)$ corresponds to the equilibrium effort of an agent with productivity level $a_{i}$. We first derive $P_{(j)}^{N}\left[e_{i} \mid e^{*}, a_{i}\right]$, the probability that agent $i$ with productivity $a_{i}$ and effort $e_{i}$ has the $j$-th highest output when all other $(N-1)$ agents exert effort based on the equilibrium effort function $e^{*}$. Because agent $i$ has no information about productivity levels of other agents, from agent $i$ 's perspective, another agent $k$ has a random productivity level $\widetilde{a}_{k}$ and a random output shock $\widetilde{\xi}_{k}$, and hence a random output $\widetilde{y}_{k}=r\left(e^{*}\left(\widetilde{a}_{k}\right)\right)+\widetilde{\xi}_{k}$. Let $F$ be the distribution function of $\widetilde{y}_{i}$, and let $f$ be the corresponding density function. It is not difficult to show that the support of $\widetilde{y}_{i}$ is $\left[\underline{s}+r\left(\underline{a} e^{*}(\underline{a})\right), \bar{s}+r\left(\bar{a} e^{*}(\bar{a})\right)\right]$ (because it can be shown that $a_{i} e^{*}\left(a_{i}\right)$ is increasing in $a_{i}$ ). We can calculate $F$ as follows:

$$
F\left(y_{i}\right)=P\left\{r\left(a_{i} e^{*}\left(\widetilde{a}_{i}\right)\right)+\widetilde{\zeta}_{i} \leq y_{i}\right\}=\int_{[\underline{a}, \bar{a}]} H\left(y_{i}-r\left(a e^{*}(a)\right)\right) g(a) d a .
$$

Let $\widetilde{y}_{(j)}^{N}$ be a random variable with cumulative distribution $F_{(j)}^{N}$ and density $f_{(j)}^{N}$ that represents the $j$-th highest value among $N$ outputs. Conditional on agent $i$ having an output shock realization $s$, the probability that agent $i$ outperforms agent $k$ by exerting effort $e_{i}$ is

$$
P\left\{r\left(a_{i} e_{i}\right)+s \geq \widetilde{y}_{k}\right\}=P\left\{r\left(a_{i} e_{i}\right)+s \geq r\left(\widetilde{a}_{k} e^{*}\left(\widetilde{a}_{k}\right)\right)+\widetilde{\zeta}_{k}\right\}=\int_{[a, \bar{a}]} H\left(r\left(a_{i} e_{i}\right)+s-r\left(a e^{*}(a)\right)\right) g(a) d a .
$$

Thus, we can write the unconditional probability that agent $i$ with productivity level $a_{i}$ has the $j$-th highest output among $N$ agents as follows:

$$
P_{(j)}^{N}\left[e_{i} \mid e^{*}, a_{i}\right]=\int_{s \in \Xi} \frac{(N-1) !}{(j-1) !(N-j) !} P\left\{r\left(a_{i} e_{i}\right)+s>\widetilde{y}_{k}\right\}^{N-j} P\left\{r\left(a_{i} e_{i}\right)+s<\widetilde{y}_{k}\right\}^{j-1} h(s) d s,
$$

because $(N-j)$ agents are ranked lower than agent $i,(j-1)$ agents are ranked higher than agent $i$, and they can be ordered in $\frac{(N-1) !}{(j-1) !(N-j) !}$ combinations. The organizer solves the following program:

$$
\begin{aligned}
\max _{N \geq K,\left(A_{(1)}, \ldots, A_{(N)}\right)} & \Pi=\sum_{j=1}^{K} \int_{\left[\underline{\underline{s}}+r\left(\underline{a} e^{*}(\underline{a})\right), \bar{s}+r\left(\bar{a} e^{*}(\bar{a})\right)\right]} y f_{(j)}^{N}(y) d y-\sum_{j=1}^{N} A_{(j)} \\
\text { s.t. } & \sum_{j=1}^{N} A_{(j)} P_{(j)}^{N}\left[e^{*}\left(a_{i}\right) \mid e^{*}, a_{i}\right]-\psi\left(e^{*}\left(a_{i}\right)\right) \geq 0 \quad \forall a_{i} \in[\underline{a}, \bar{a}] \\
& e^{*}\left(a_{i}\right)=\arg \max _{e_{i} \in \mathbb{R}_{+}} \sum_{j=1}^{N} P_{(j)}^{N}\left[e_{i} \mid e^{*}, a_{i}\right] A_{(j)}-\psi\left(e_{i}\right) \quad \forall a_{i} \in[\underline{a}, \bar{a}] .
\end{aligned}
$$

The objective of the organizer given in (6) is to choose $N(\geq K)$ and $\left(A_{(1)}, \ldots, A_{(N)}\right)$ that maximize his expected profit. Participation constraint (7) guarantees that each agent receives non-negative from the contest in equilibrium, and hence chooses to participate in the contest. Constraint (8) is the incentive compatibility constraint through which the organizer considers the agent's utility maximization problem. In this problem, 
each agent $i$ with productivity $a_{i}$ chooses an effort $e_{i}$ that maximizes the expected prize $\sum_{j=1}^{N} P_{(j)}^{N}\left[e_{i} \mid e^{*}, a_{i}\right] A_{(j)}$ less the expected cost $\psi\left(e_{i}\right)$, assuming that every other agent chooses an effort based on the function $e^{*}$ in equilibrium.

Discussion The present model framework encompasses the main features of models used in the innovation contest literature as detailed in Table 1. This framework includes both heterogeneous agents and output shocks that affect agents' outputs as well as the organizer's payoff. Unfortunately, without making very restrictive assumptions, such a generic model has limited analytical tractability for two reasons. First,

$f_{(j)}^{N}\left(y_{i}\right)=\frac{N !}{(j-1) !(N-j) !}\left(1-F\left(y_{i}\right)\right)^{j-1} F\left(y_{i}\right)^{N-j} f\left(y_{i}\right)$ expression in the organizer's objective (6) is highly complex because it contains the multiplication of $N$ integrals stemming from of the distribution $F$ in (4), and its density $f$. Second, the distribution $F$ in (4) depends on the equilibrium effort $e^{*}$, so one needs to characterize the agent's equilibrium effort $e^{*}$ solving the agent's problem in (8) before optimizing the organizer's decisions. Yet, in (8), the agent's probability of attaining rank $j, P_{(j)}^{N}\left[e_{i} \mid e^{*}, a_{i}\right]$, is highly complex, and so is the system of equations arising from agents' first-order conditions. Due to these technical complications, most papers in the literature have chosen one of two pathways: either focus on agents' uncertainty by suppressing their heterogenous ability levels or focus on agents' heterogeneity by suppressing the uncertainty they face. Accordingly, we use this separation while discussing the literature in the following section, and we analyze these two analytically tractable special cases separately in $\S 4$ and $\S 5$.

\section{A Brief Taxonomy of Contest Literature}

In this section, we briefly discuss contest literature in general, and then discuss the distinguishing factors of innovation contests. Although there is a stream of empirical studies on contests, we restrict attention to theoretical work.

The research on contests is not new. Following the pioneering works of Tullock $(1967,1980)$ and Lazear and Rosen (1981), contests have been used in various settings such as labor tournaments (e.g., Green and Stokey 1983, Nalebuff and Stiglitz 1983) in which employers aim to incentivize employees to exert more effort, and sales contests (e.g., Kalra and Shi 2001) in which firms elicit effort from salespeople. Several topics have been explored such as the optimal set of awards that an organizer should distribute (e.g., Moldovanu and Sela 2001, Kalra and Shi 2001), the risk-taking behavior of agents in a contest (Hvide 2002), having multiple rounds or a single round in a contest (Moldovanu and Sela 2006), and designing auction-based mechanisms in which heterogeneous agents have different costs (Che and Gale 2003, Siegel 2009). Vojnović (2015) provides a detailed overview of such contests. Different from these classical contests, innovation contests possess two important distinct features: (i) an organizer is interested in only the best solution(s) rather than all solutions (i.e., $K<N$ ) and (ii) agents' uncertainty impacts an organizer's profit from a contest, and hence the organizer considers agents' uncertainty as well as their effort while determining contest rules. 
Table 1 Review of Innovation Contest Literature that Use a Variant of the Present Model Framework.

\begin{tabular}{|c|c|c|c|}
\hline Paper & Model of Uncertainty & Model of Heterogeneity & Other Features \\
\hline $\begin{array}{l}\text { Terwiesch and } \\
\mathrm{Xu}(2008)\end{array}$ & $\begin{array}{l}\text { (i) Trial-and-error projects with } \\
\text { no improvement effort and no } \\
\text { taste shock; (ii) ideation projects } \\
\text { with no trial effort and a } \\
\text { Gumbel distributed taste shock }\end{array}$ & $\begin{array}{l}\text { Expertise-based projects with } \\
\text { heterogeneous expertise } \\
\text { levels and no uncertainty }\end{array}$ & $\begin{array}{l}\text { The organizer's payoff is in the } \\
\text { weighted combination of the best } \\
\text { output and the average of all outputs }\end{array}$ \\
\hline Ales et al. (2016) & $\begin{array}{l}\text { A model that utilizes Lemma } \\
1(a) \text {, and assumes a log-concave } \\
\text { or increasing density for the } \\
\text { output shock }\end{array}$ & Homogenous agents & $\begin{array}{l}\text { A general utility function for the } \\
\text { organizer that allows risk aversion } \\
\text { and complementarity }\end{array}$ \\
\hline $\begin{array}{l}\text { Mihm and } \\
\text { Schlapp (2016) }\end{array}$ & $\begin{array}{l}\text { Ideation projects with no } \\
\text { trial-and-error experiments and } \\
\text { uniformly distributed taste } \\
\text { shock }\end{array}$ & $\begin{array}{l}\text { Expertise-based } \\
\text { heterogeneity in the second } \\
\text { period with feedback }\end{array}$ & $\begin{array}{l}\text { A two-period model with two agents } \\
\text { where feedback can be given to } \\
\text { agents between periods }\end{array}$ \\
\hline $\begin{array}{l}\text { Nittala and } \\
\text { Krishnan (2016) }\end{array}$ & $\begin{array}{l}\text { Ideation projects as in Terwiesch } \\
\text { and } \mathrm{Xu}(2008) \text { with Gumbel } \\
\text { distributed taste shock }\end{array}$ & Homogenous agents & $\begin{array}{l}\text { Internal contests where the organizer } \\
\text { incurs a cost from agents' efforts; and } \\
\text { external contests where there is a risk } \\
\text { for linkage of intellectual property }\end{array}$ \\
\hline $\begin{array}{l}\text { Körpeoğlu and } \\
\text { Cho (2017) }\end{array}$ & No output shock & $\begin{array}{l}\text { vity-based projects } \\
\text { mpass cost-projects } \\
\text { rtise-based projects }\end{array}$ & $\begin{array}{l}\text { The same profit function for the } \\
\text { organizer as T\&X; fixed cost of entry } \\
\text { when driving equilibrium }\end{array}$ \\
\hline Ales et al. (2017) & $\begin{array}{l}\text { Same model of uncertainty with } \\
\text { the present chapter }\end{array}$ & Homogenous agents & No other features \\
\hline $\begin{array}{l}\text { Hu and Wang } \\
(2017)\end{array}$ & $\begin{array}{l}\text { A model that utilizes Lemma } \\
1(\mathrm{a}) \text {, and assumes a symmetric } \\
\text { log-concave density for the } \\
\text { output shock }\end{array}$ & $\begin{array}{l}\text { Two agents model where } \\
\text { each agent has high ability in } \\
\text { exactly one of two attributes }\end{array}$ & $\begin{array}{l}\text { Two attribute model with the option } \\
\text { of running one contests per each } \\
\text { attribute or a single contest for both } \\
\text { attributes }\end{array}$ \\
\hline $\begin{array}{l}\text { Körpeoğlu et al. } \\
\text { (2017) }\end{array}$ & $\begin{array}{l}\text { A model that utilizes Lemma } \\
\text { 1(a) and assumes a log-concave } \\
\text { density for the output shock }\end{array}$ & Homogenous agents & $\begin{array}{l}\text { Multiple contest organizers and a } \\
\text { more general cost function that } \\
\text { allows economies of scope across } \\
\text { different contests }\end{array}$ \\
\hline $\begin{array}{l}\text { Stouras et al. } \\
\text { (2017) }\end{array}$ & $\begin{array}{l}\text { A common taste shock that does } \\
\text { not affect agents' relative rank } \\
\text { but impacts their absolute } \\
\text { outputs }\end{array}$ & $\begin{array}{l}\text { Heterogeneous expertise } \\
\text { levels }\end{array}$ & $\begin{array}{l}\text { Fixed cost of entry leading to } \\
\text { uncertain number of participants }\end{array}$ \\
\hline
\end{tabular}

As discussed in $\S 2$, due to tractability issues, the literature on innovation contests has been divided into two streams. The first stream focuses primarily on innovation contests in which agents exert effort or conduct random trials when their outcomes are uncertain, while suppressing agent heterogeneity. Terwiesch and Xu (2008) show that agents' efforts always decrease with more participants but an open contest is always optimal when considering agents' Gumbel distributed shocks. Ales et al. (2016) show that more agents may lead to increased or decreased effort from agents depending on the distribution $H$ of the output shock, and further show that an open contest is optimal for a general distribution only when the output shock distribution is sufficiently spread out or the organizer seeks many diverse solutions. Meanwhile, Ales et al. (2017) characterize the optimal set of awards in this environment, and prove that when agents' uncertainty has a log-concave or increasing density function, the winner-take-all award scheme is optimal. Mihm and Schlapp (2016) compare different types of feedback (e.g., public, private, or no feedback) that can be used to improve the contest outcome. Nittala and Krishnan (2016) compare internal innovation contests within firms, in which the organizer incurs a cost from agents' efforts (as they are employees), with external contests where the organizer utilizes independent agents. Hu and Wang (2017) study a case where 
the organizer seeks two attributes, and compare running a single contest for both attributes with running two contests - one for each attribute. Körpeoğlu et al. (2017) study multiple contests tackled by the same set of agents, and show that when organizers seek innovative solutions rather than low-novelty tasks, it may be better for organizers to allow agents to freely participate in multiple contests rather than to restrict them to a single contest. They further characterize the optimal number of parallel contests, and show that this optimal number increases with the novelty of the solutions organizers seek.

A second stream of the literature studies contests in which heterogeneous agents compete but with no uncertainty in agents' outputs. These papers build on prior research in economics such as Moldovanu and Sela (2001), who analyze the optimal set of awards in a cost-based model where agents are heterogeneous in their cost of effort. Liu et al. (2007) use a similar model to Moldovanu and Sela (2001) to study prize structure, segmentation, and handicapping in a consumer contest where the organizer aims to stimulate consumption of a good. Terwiesch and $\mathrm{Xu}$ (2008) analyze an expertise-based model in which agents are heterogeneous in their initial expertise, and show that an open contest can be optimal under certain conditions. Körpeoğlu and Cho (2017) propose an alternative productivity-based model to unify cost-based model of Moldovanu and Sela (2001) and expertise-based model of Terwiesch and Xu (2008). They show that an agent's equilibrium effort can increase with more participants, and offer a precise explanation to this result by detailing two opposing drivers. Körpeoğlu and Cho (2017) further show that an open contest is more likely to be optimal than what prior studies asserted. Recently, Stouras et al. (2017) analyze how an organizer can promote agents' participation and effort when only a random number of agents participate in the contest because agents incur large fixed costs of entry, which discourage some agents from participating.

Besides the two streams of research on innovation contests discussed above, there are some papers that use a different, more tailored modelling framework to study special types of innovation contests. Taylor (1995) considers a contest among a pool of identical agents, in which each agent conducts random trials until the best output of those trials reaches a pre-determined quality level. Fullerton and McAfee (1999) analyze a contest in which an organizer auctions entry into a contest. Both of these papers show that more agents in a contest leads to a lower equilibrium effort for every agent, but unlike Terwiesch and $\mathrm{Xu}$ (2008) and Ales et al. (2016), these papers conclude that the organizer should restrict entry to the contest. Erat and Krishnan (2012) study design contests in which each agent selects one design approach among a finite set of approaches. Bimpikis et al. (2016) study information extraction and disclosure strategies that keep agents active in dynamic contests.

\section{Contests with Uncertainty}

In this section, we analyze innovation contests where the output uncertainty plays a larger role than the heterogeneity of agents. To implement this, we suppress the agent heterogeneity by setting $\bar{a}=\underline{a}=1$. With this assumption, in a symmetric equilibrium, each agent exerts the same equilibrium effort $e^{*}\left(a_{i}\right)=e^{*}$. In 
this case, we can simplify agent $i$ 's probability of producing the $j$-th highest output in (5) as follows:

$$
P_{(j)}^{N}\left[e_{i} \mid e^{*}\right]=\int_{s \in \Xi} \frac{(N-1) !}{(j-1) !(N-j) !} H\left(s+r\left(e_{i}\right)-r\left(e^{*}\right)\right)^{N-j}\left(1-H\left(s+r\left(e_{i}\right)-r\left(e^{*}\right)\right)\right)^{j-1} h(s) d s .
$$

Then, we can rewrite the organizer problem (6)-(8) as follows:

$$
\begin{aligned}
\max _{N \geq K,\left(A_{\left.(1), \ldots, A_{(N)}\right)}\right.} \Pi=K r\left(e^{*}\right)+E\left[\sum_{j=1}^{K} \widetilde{\xi}_{(j)}^{N}\right]-\sum_{j=1}^{N} A_{(j)} \\
\text { s.t. } \frac{1}{N} \sum_{j=1}^{N} A_{(j)} \geq \psi\left(e^{*}\right) \\
e^{*}=\arg \max _{e_{i} \in \mathbb{R}_{+}} \sum_{j=1}^{N} P_{(j)}^{N}\left[e_{i} \mid e^{*}\right] A_{(j)}-\psi\left(e_{i}\right) .
\end{aligned}
$$

In $\S 4.1$, we analyze the optimal award scheme, and in $\S 4.2$ we study the decision of the organizer to restrict entry or not.

\subsection{Optimal Award Scheme}

This section discusses the optimal award scheme based on Section 3 of Ales et al. (2017). As discussed in $\S 2$, a tournament organizer determines an award scheme by choosing a set of prizes $\left(A_{(1)}, A_{(2)}, \ldots, A_{(N)}\right)$ for each ranked agent. It is common in the literature to focus on environments where the WTA is used. However, the WTA scheme may not always be optimal. To examine when the WTA scheme is justified, Ales et al. (2017) derive a necessary and sufficient condition in their Proposition 1 under which the WTA scheme is optimal. Specifically, they link the optimality of the WTA scheme to (i) the distribution of the output shock and (ii) whether the participation constraint (11) is satisfied. Without going into details about this condition, we will discuss when it is violated and when it is satisfied.

Proposition 1. (Propositions 2 of Ales et al. 2017) For any given A, the winner-takes-all (WTA) award scheme is suboptimal when one of the following conditions is satisfied:

(i) $\lim _{s \rightarrow \bar{s}} h(s)=0, \lim _{s \rightarrow \bar{s}}\left|\frac{h^{\prime}(s)}{h(s)}\right|<\infty$, and

$$
\int_{s \in \Xi}\left[H_{(j)}^{N}(s)-H_{(1)}^{N}(s)\right]\left(\frac{h^{\prime}(s)}{h(s)}\right)^{\prime} d s>0,
$$

where (13) holds if $h(s)$ is strictly log-convex (i.e., $d^{2} \log h(s) / d s^{2}>0 \forall s$ ).

(ii) $\frac{A}{N}-\psi\left(\left(\frac{\psi^{\prime}}{r^{\prime}}\right)^{-1}\left(A \int_{s \in \Xi}(N-1) H(s)^{N-2} h(s)^{2} d s\right)\right)<0$.

We first discuss condition (i), using an example that satisfies this condition. Observe that the density $h$ in Figure 1(a) features a large highly convex and decreasing region between its peak point and its fat right tail. In this example, an agent's effort may be more effective in increasing the agent's probability of attaining some rank $j(>1)$ than that of becoming the winner. Thus, reducing the winner prize $A_{(1)}$ and increasing award $A_{(j)}$ corresponding to this rank $j$ makes the agent's effort more effective to win a prize, and hence 


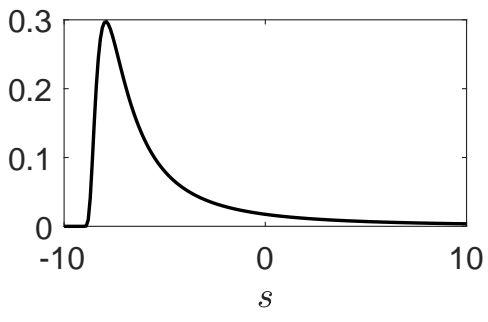

(a) WTA scheme is suboptimal.

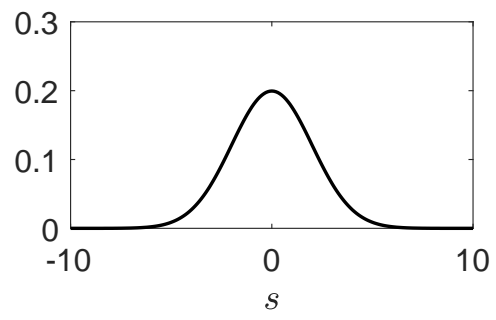

(b) WTA scheme is optimal.

Fig. 1 (a) Frechet density with mean 0 , shape parameter $\beta=1.2$, and scale parameter $\mu=1.5$ and (b) Gumbel density with mean 0 and $\mu=1$, which is log-concave.

the agent finds it optimal to increase effort. In practice, this may occur when it is likely that most agents generate low outputs while a few agents generate very high outputs in the contest; for example, when agents' outputs are evaluated based on popularity among consumers and only few solutions are expected to be extremely popular (e.g., evaluation based on download counts for apps in the 2012 Samsung Smart App Challenge).

Condition (ii) in Proposition 1 specifies when agents do not find it beneficial to participate a contest under the WTA scheme. In this case, the participation constraint (11) is violated under the solution to the agent's problem in (12), because each agent's effort in equilibrium is too high in a WTA contest to be justified by the expected winner prize. In this case, the WTA scheme cannot be optimal to the organizer because there is no equilibrium under the WTA. Thus, the organizer may offer multiple awards to strategically reduce agents' effort in order to guarantee their participation. Ales et al. (2017) show that the condition in Proposition 1(ii) holds when agents' uncertainty is sufficiently low and/or their cost function $\psi$ has low convexity. This suggests that, all else being equal, the WTA scheme is more likely to be optimal in a contest that seeks highly technical or innovative solutions that demand more substantial increase in agents' marginal costs of effort (e.g., an RTP challenge at InnoCentive).

Next, we discuss sufficient conditions for $\widetilde{\xi}_{i}$ under which the WTA scheme is optimal.

Proposition 2. (Proposition 3 of Ales et al. 2017) Suppose that (11) holds under the WTA award scheme. Then, for any given $A$, the WTA award scheme is optimal when the density $h(s)$ of the output shock $\widetilde{\xi}_{i}$ is log-concave (i.e., $\left.d^{2} \log h(s) / d s^{2} \leq 0 \forall s\right)$ or increasing in $s$.

According to Proposition 2, the WTA award scheme is optimal when the output shock density is logconcave or increasing. When a density function is log-concave or increasing, no portion of its support is highly convex and decreasing (e.g., see Figure 1(b)), and hence such a density violates (13) in Proposition 1. In fact, the class of distributions proposed in Proposition 2 is fairly large because many of the commonlyused distributions are either log-concave (e.g., Gumbel, exponential, normal, uniform, and logistic distributions, and Weibull distribution with a shape parameter greater than 1) or increasing (e.g., Weibull distribution with a shape parameter less than or equal to 1). Thus, in practice, the WTA scheme may be appropriate 
in contests where homogenous agents expect that their outputs will be evenly distributed rather than a few agents generating very high outputs.

\subsection{Open Innovation and Agents' Incentives}

In this section, we build our discussion on Section 4 of Ales et al. (2016) that shows when the organizer should hold an open contest that allows entry of all agents who wish to participate in the contest. The number of participants $N$ directly impacts the organizer's profit $\Pi=K r\left(e^{*}\right)+E\left[\sum_{j=1}^{K} \widetilde{\xi}_{(j)}^{N}\right]-A$ in two ways. First, $N$ affects the agent's equilibrium effort $e^{*}$ and hence $K r\left(e^{*}\right)$, since $K$ is fixed and $r(\cdot)$ is increasing. Second, $N$ affects $E\left[\sum_{j=1}^{K} \widetilde{\xi}_{(j)}^{N}\right]$, which represents the expected value of the best $K$ outcomes from $N$ random shocks. It is easy to see that this term increases with $N(\geq K)$ for any $K$ because a more diverse set of solutions increases the expected value of the best $K$ outputs. Thus, for a given award $A$, depending on how $e^{*}$ changes with $N, \Pi$ can increase or decrease with $N$. When $\Pi$ is increasing with $N$, it is optimal for the organizer to choose an open contest. In the remainder of this section, we first study how the agent's equilibrium effort $e^{*}$ changes with $N$, and then when the organizer should choose an open contest.

As the number of participants $N$ increases, one may expect that agents would decrease their effort $e^{*}$ because their individual chance of becoming the winner decreases. Yet, Ales et al. (2016) show, counter-intuitively, that more participants do not always induce lower efforts from agents. To discuss this finding, we can derive the equilibrium effort $e^{*}$ using the condition $\psi^{\prime}\left(e^{*}\right) / r^{\prime}\left(e^{*}\right)=A I_{N}$, where $I_{N} \equiv \int_{s \in \Xi}(N-1) H(s)^{N-2} h(s)^{2} d s$. Because $\psi^{\prime} / r^{\prime}$ is increasing, the effort $e^{*}$ is increasing (resp., decreasing) in $N$ whenever $I_{N}$ is increasing (resp., decreasing) in $N$; see the following example for illustration.

Example 1. (i) When $\widetilde{\zeta}_{i}$ follows a Weibull distribution with mean 0 , shape parameter $\beta=1$, and scale parameter $\mu$, we have $I_{N}=\frac{N-1}{\mu N}$ increasing in $N$. Thus, $e^{*}$ increases with $N$ as well.

(ii) When $\widetilde{\zeta}_{i}$ follows a Gumbel distribution with mean 0 and scale parameter $\mu$, we have $I_{N}=\frac{N-1}{\mu N^{2}}$. In this case, $I_{N}$ is decreasing in $N$, and so is $e^{*}$.

Ales et al. (2016) explain the intuition for why more participants can increase the equilibrium effort $e^{*}$ by analyzing $I_{N}$ as follows. From (12), the agent's marginal benefit of increasing effort is $A\left(P_{(1)}^{N}\right)^{\prime}\left[e^{*} \mid e^{*}\right]=$ $A r^{\prime}\left(e^{*}\right) I_{N}$, and it increases with $\left(P_{(1)}^{N}\right)^{\prime}\left[e^{*} \mid e^{*}\right]=r^{\prime}\left(e^{*}\right) I_{N}$, which represents a marginal change of the winning probability with additional effort. Thus, how $e^{*}$ changes with $N$ depends not on the winning probability but on the marginal impact of additional effort on the winning probability. When $I_{N+1}>I_{N}$, more intense competition due to a larger number of agents increases the marginal benefit of an agent's additional effort on the probability of winning. In this case, agents increase effort when faced with more intense competition.

Building on this observation, we next presents a necessary and sufficient condition on the output shock $\widetilde{\xi}_{i}$ under which the equilibrium effort $e^{*}$ decreases with the number agents $N$, and presents sufficient conditions under which $e^{*}$ increases with $N$. 
Proposition 3. (Proposition 1 in Ales et al. 2016) (a) The equilibrium effort $e^{*}$ is non-increasing for any $N \geq 2$ if and only if the density $h(s)$ of the output shock $\widetilde{\xi}_{i}$ satisfies

$$
\int_{s \in \Xi}(1-H(s)) H(s) h^{\prime}(s) d s \leq 0 .
$$

(b) Suppose $h(s)$ is increasing in sor the symmetric function of $h$ with respect to $y$-axis, i.e., $h_{r}(s) \equiv h(-s)$ for all s, satisfies (14) strictly. Then, $e^{*}$ is increasing up to some $N^{*}$ (where $N^{*}=\infty$ for increasing $h$ ).

Condition (14) in Proposition 3(a) ensures that the density $h$ is sufficiently right-skewed as in Example 1(ii), and this condition is satisfied by any symmetric log-concave density (e.g., normal, logistic) as well as Gumbel and exponential densities. This implies that when agents believe that a bad outcome is at least as likely as a good outcome, they tend to decrease effort with more participants. On the other hand, whenever the necessary and sufficient condition given in (14) is violated, Proposition 3(b) shows that the equilibrium effort $e^{*}$ is increasing in $N$ up to some $N^{*}$. For example, this condition is violated by a reversed Gumbel distribution or a Weibull distribution. This implies that when agents expect good outcomes with high likelihood, they tend to increase effort with more participants in the contest. This finding is supported from experimental results of List et al. (2014), which demonstrate that in contests with small size, when agents know that they have a high chance of getting favorable outcomes, increasing the number of participants may have positive impact on agents' efforts (see Ales et al. 2016 for detailed discussion).

We next discuss the findings of Ales et al. (2016) about when the organizer should hold an open contest that allows all agents who wish to participate in the contest to do so. When the equilibrium effort $e^{*}$ is increasing in the number of agents $N$, the organizer's profit increases with $N$ because more participants in the contest also provide a more diverse set of solutions to the organizer (i.e., increases $\sum_{j=1}^{K} E\left[\widetilde{\xi}_{(j)}^{N}\right]$ as discussed above). Thus, it is optimal for the organizer to hold an open contest.

When the equilibrium effort $e^{*}$ is decreasing in the number of agents $N$, the organizer's profit may increase or decrease with $N$, depending on whether the benefit of having a diverse set of solutions outweighs the agents' reduced effort. To quantify the benefit of having a more diverse set of solutions for a general output shock distribution $H(s)$, the notion of a scale transformation is used. When the output shock $\widetilde{\xi}_{i}$ is transformed with scale parameter $\alpha$, the transformed output shock (i.e., $\widehat{\xi}_{i}=\alpha \widetilde{\xi}_{i}$ ) has the same mean as $\widetilde{\xi}_{i}$ at 0 , and its variance is $\alpha^{2}$ times the variance of $\widetilde{\xi}_{i}$. When $\alpha>1, \widehat{\xi}_{i}$ has a larger variance and its density is more spread out. The following proposition of Ales et al. (2016) shows that when the output shock density $h$ is sufficiently spread out, an open contest is optimal.

Proposition 4. (Proposition 2 of Ales et al. 2016) For any distribution $H$ of the output shock $\widetilde{\xi}_{i}$, there exist $\bar{\alpha}$ such that under a scale transformation of $\widetilde{\xi}_{i}$ with $\alpha \geq \bar{\alpha}$, an open contest with unrestricted entry is optimal for any number of contributors $K$. 
Proposition 4 shows that when the agents' output uncertainty is sufficiently large, an open contest is optimal. In practice, agents can face large uncertainty when the organizer seeks innovative solutions (e.g., writing a software that matches 3D objects with 2D images) rather than low-novelty tasks (e.g., findings bugs in a software). Similarly, how broadly the organizer's problem is defined or how objective the evaluation criteria are can play a role in agents' uncertainty. Overall, Proposition 4 shows that open innovation initiatives are justified when the organizer seeks innovative solutions for broadly defined problems and/or with subjective judging criteria.

Ales et al. (2016) further show in their Proposition 3 that the threshold scale parameter $\bar{\alpha}$, which is the minimum $\alpha$ required for an open contest, decreases with the number of contributors $K$. This suggests that an open contest is more likely to be optimal when there are more contributors. This result, in conjunction with Proposition 4, generates insights that are consistent with practice. For example, Samsung Smart App Challenge and Goldcorp Challenge are open contests, probably because agents face large uncertainty, and anticipate a large number of contributors. On the other hand, in the design contest for the official emblem of the 2014 FIFA World Cup, participating agencies were restricted to 25 (James 2014). Although this contest also involves uncertainty, the restricted entry may be because there is a single contributor.

\section{Contests with Heterogenous Agents}

In this section, we go back to our general model, and analyze contests where agents feature heterogenous productivity levels, while suppressing agents' uncertainty. This model may be suitable for contests in which agents engage in low-novelty tasks, and their ability levels are highly heterogeneous. For ease of illustration, we focus on a case with a single contributor (i.e., $K=1$ ) and a linear cost of effort $\psi\left(e_{i}\right)=c e_{i}$. This model corresponds to a special case of Körpeoğlu and Cho (2017) by assuming that the organizer is interested in only the best solution.

In a symmetric equilibrium, an agent with productivity level $a_{i}$ chooses an effort level according to the equilibrium effort function $e^{*}\left(a_{i}\right)$, and creates an output $y^{*}\left(a_{i}\right)$. In this case, each agent can decide on an output level $y_{i}$ by choosing an appropriate effort $e_{i}=r^{-1}\left(y_{i}\right) / a_{i}$ because $y_{i}=r\left(a_{i} e_{i}\right)$. Since agent $i$ does not know other agents' ability levels, the equilibrium output $\widetilde{y}^{*}=y^{*}(\widetilde{a})$ is uncertain, where $\widetilde{a}$ is a random variable that represents another agent's unknown productivity level. Assuming that $y^{*}$ is an increasing function of a productivity level (verified later), we can write the probability that agent $i$ is better than another agent as $P\left(y_{i} \geq \widetilde{y}^{*}\right)=G\left(\left(y^{*}\right)^{-1}\left(y_{i}\right)\right)$. Thus, each agent $i$ 's problem in (8) can be rewritten as

$$
\max _{y_{i}} \sum_{j=1}^{N} A_{(j)} \frac{(N-1) !}{(j-1) !(N-j) !} G\left(\left(y^{*}\right)^{-1}\left(y_{i}\right)\right)^{N-j}\left(1-G\left(\left(y^{*}\right)^{-1}\left(y_{i}\right)\right)\right)^{j-1}-c r^{-1}\left(y_{i}\right) / a_{i} .
$$

In equilibrium, $y_{i}=y^{*}\left(a_{i}\right)$ for all agents with productivity $a_{i}$. Thus, for agent $i$ to participate, the utility from the contest must be non-negative; i.e., 


$$
\sum_{j=1}^{N} A_{(j)} \frac{(N-1) !}{(j-1) !(N-j) !} G\left(a_{i}\right)^{N-j}\left(1-G\left(a_{i}\right)\right)^{j-1}-c r^{-1}\left(y^{*}\left(a_{i}\right)\right) / a_{i} \geq 0 .
$$

Lastly, given the equilibrium effort $e^{*}\left(a_{i}\right)=r^{-1}\left(y^{*}\left(a_{i}\right)\right) / a_{i}$, the organizer's profit in (6) becomes:

$$
\Pi=\int_{\underline{a}}^{\bar{a}} r\left(a_{i} e^{*}\left(a_{i}\right)\right) g_{(1)}^{N}\left(a_{i}\right) d a_{i}-A .
$$

In $\S 5.1$ we study the optimal award scheme, and in $\S 5.2$ we analyze the decision of the organizer to hold an open contest or restrict entry to the contest.

\subsection{Optimal Award Scheme}

In this section, as in $\S 4.1$, we discuss when the WTA award scheme is optimal. The result of this section is new, so its proof is presented in Appendix. Suppose that the organizer distributes two prizes to the winner and the runner-up with a total prize of $A$. (The analysis can easily be generalized to multiple prizes.) Let $\alpha \in[0,0.5]$ be a proportion of the total prize that is awarded to the runner-up. Then., the winner prize $A_{(1)}=(1-\alpha) A$ and the runner-up prize $A_{(2)}=\alpha A$. To investigate when the WTA (i.e., $\alpha=0$ ) is optimal, we use a specific functional form for the effort function $r(e)=\theta \frac{e^{1-b}-1}{1-b}$ (where $b \geq 0$ ), which is a Constant Relative Risk Aversion (CRRA) function. The CRRA effort function collapses to the linear effort function of Moldovanu and Sela (2001) and Mihm and Schlapp (2016) when $b=0$ (i.e., $\lim _{b \rightarrow 0} \theta \frac{e^{1-b}-1}{1-b}=\theta e$ ), and to the logarithmic effort function of Terwiesch and $\mathrm{Xu}$ (2008) when $b=1$ (i.e., $\lim _{b \rightarrow 1} \theta \frac{e^{1-b}-1}{1-b}=\theta \log e$ ).

Proposition 5. Let $r(e)=\frac{e^{1-b}-1}{1-b}$. There exists $b_{0}$ such that for all $b \leq b_{0}$, it is optimal for the organizer to set $\alpha=0$. In contrast, when $\int_{\underline{a}}^{\bar{a}} a_{i}\left(a_{i} e^{*}\left(a_{i}\right)\right)^{-b} \frac{\partial e^{*}\left(a_{i}\right)}{\partial \alpha} g_{(1)}^{N}\left(a_{i}\right) d a_{i}>0$, it is optimal for the organizer to set $\alpha>0$.

Proposition 5 shows that the WTA scheme is optimal when the concavity of the effort function (captured in parameter $b$ ) is small; this result is also illustrated in Figures 2(a)-(b). In contrast, as Figure 2(c) depicts, when $b$ is large, the organizer's profit improves by increasing the weight on the second prize, so the WTA scheme is suboptimal. To understand the intuition behind this result, we need to analyze the derivative of the organizer's profit with respect to the weight on the second prize $\alpha$ :

$$
\frac{\partial \Pi}{\partial \alpha}=\theta \int_{\underline{a}}^{\bar{a}} a_{i}\left(a_{i} e^{*}\left(a_{i}\right)\right)^{-b} \frac{\partial e^{*}\left(a_{i}\right)}{\partial \alpha} g_{(1)}^{N}\left(a_{i}\right) d a_{i} .
$$

As the organizer increases the weight on the second prize, agents with low productivity increase effort (i.e., $\frac{\partial e^{*}\left(a_{i}\right)}{\partial \alpha}>0$ for small $a_{i}$ ) and agents with high productivity reduce effort (i.e., $\frac{\partial e^{*}\left(a_{i}\right)}{\partial \alpha}<0$ for large $a_{i}$ ). There are two forces that determine whether the former effect or the latter effect dominates. On the one hand, because the organizer is interested in the best output, the organizer has larger weight on the effort of the highproductivity agents than low-productivity agents. On the other hand, because the equilibrium output $y^{*}\left(e_{i}\right)$ is increasing in $a_{i}$, and the effort function $r$ is concave, additional effort by low-productivity agents leads to larger increase in their outputs (i.e., $\left(a_{i} e^{*}\left(a_{i}\right)\right)^{-b}$ is decreasing in $a_{i}$ ). When the effort function is linear or close to linear (i.e., $b$ is small), the negative effect of the second prize (i.e., $\alpha A$ ) on the equilibrium effort of 


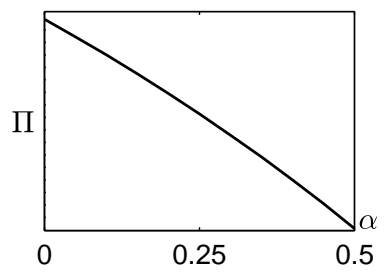

(a) $b=0.5$

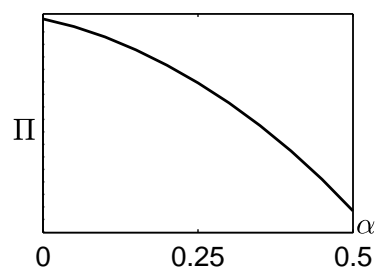

(b) $b=1$

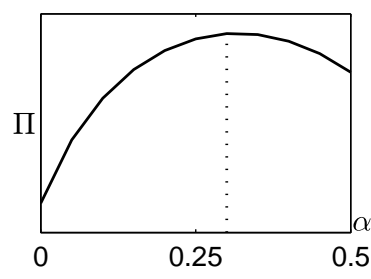

(c) $b=1.5$

Fig. 2 The organizer's profit $\Pi$ as a function of the weight on the runner-up prize $\alpha$ when $\widetilde{a} \sim \operatorname{Uniform}(0,1), N=10, r(e)=$ $\frac{e^{1-b}}{1-b}, A=1$, and $c=0.1$.

high-productivity agents outweighs its positive effect on the equilibrium effort of low-productivity agents, so the WTA scheme is optimal. When the effort function is highly concave (i.e., $b$ is large), additional effort by low-productivity agents leads to significant increase in their outputs so it is optimal for the organizer to offer a second prize.

Proposition 5 is similar to Propositions 2 and 5 of Moldovanu and Sela (2001), who study a cost-based heterogeneity model with $r\left(e_{i}\right)=e_{i}$, and all contributors (i.e., $K=N$ ). Their Proposition 2 shows that the WTA scheme is optimal when the cost of effort $\psi$ is linear. Proposition 5 in Moldovanu and Sela (2001) assumes convex $\psi$, and proposes a necessary and sufficient condition for the WTA scheme to be suboptimal. Our Proposition 5 extends their results to the productivity-based model where the organizer is interested in the best solution (i.e., $K=1$ ), and shows that the concavity of the effort function $r$ is another factor that affects the optimality of the WTA scheme.

\subsection{Open Innovation and Agents' Incentives}

In this section, we discuss when the organizer should allow the entry of all agents who wish to participate in a winner-take-all contest. We build on Section 3 of Körpeoğlu and Cho (2017). As in §4.2, we first discuss how the equilibrium output changes with the number of agents in the contest. Then, we present an original result regarding the impact of the number of agents on the organizer. Before describing how the equilibrium effort and output change with the number of agents $N$, we present the following result from Körpeoğlu and Cho (2017). The lemma characterizes the equilibrium effort and output under the WTA scheme, while generalizing this lemma to the case with multiple awards in Lemma A1 of Appendix.

Lemma 2. (Lemma 1 of Körpeoğlu and Cho 2017) In a productivity-based project with a general productivity distribution $G$ and a general effort function $r$, an agent with productivity $a_{i}$ has equilibrium effort $e^{*}\left(a_{i}\right)=$ $\frac{A}{c a_{i}} \int_{\underline{a}}^{a_{i}} a g_{(1)}^{N-1}(a)$ da and equilibrium output $y^{*}\left(a_{i}\right)=r\left(\frac{A}{c} \int_{\underline{a}}^{a_{i}} a g_{(1)}^{N-1}(a) d a\right)$.

We discuss how the agent's output $y^{*}$ and effort $e^{*}$ change with the number of agents $N$, by contrasting the implications of our model with empirical observations. Let $y^{*, N}$ and $e^{*, N}$ denote the agent's output and effort, respectively, when there are $N$ agents in the contest. Figure 3(a), adapted from Figure 7 of Boudreau et al. (2012), depicts how the agent's output changes with an additional high-ability "superstar" (dotted 

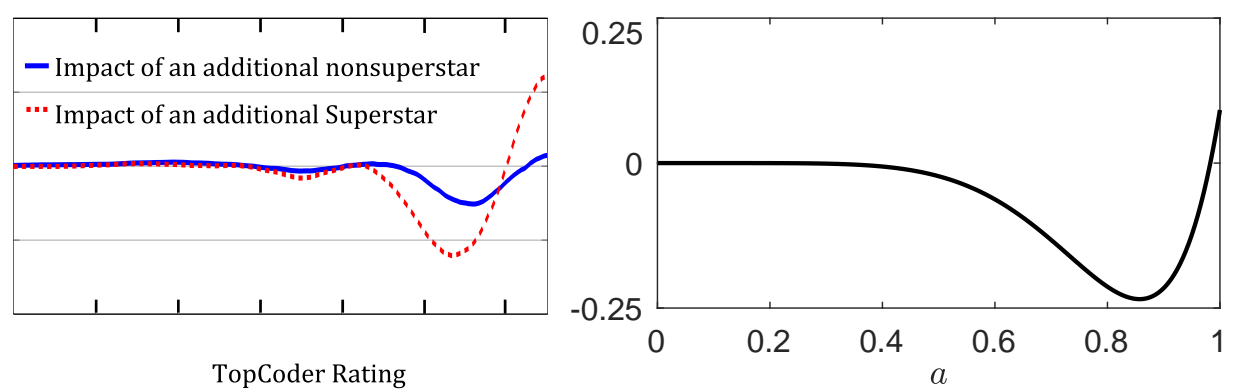

Fig. 3 The impact of an additional agent on the agent's output: (a) empirical observation in Boudreau et al. (2012), and (b) our theoretical prediction of $y^{*, N+1}-y^{*, N}$ when $G \sim$ Beta with parameters 1 and $0.75 ; N=10, r(e)=\frac{e^{0.9}}{0.9}, A=1$, and $c=0.1$.

curve) or an additional lower-ability "non-superstar" (normal curve) in software development contests organized by TopCoder. For both cases, an additional agent has a minimal effect on low-ability agents with TopCoder rating less than 2000, whereas it has a negative effect on moderate-ability agents with TopCoder rating between 2000 and 2400, and it has a positive effect on high-ability agents with TopCoder rating over 2400. To compare such empirical observation with our theoretical prediction, we illustrate the impact of an additional agent on the output of agents with different productivity levels in Figure 3(b) by plotting $y^{*, N+1}\left(a_{i}\right)-y^{*, N}\left(a_{i}\right)$ over $a_{i}$. One can clearly see that the patterns in Figure 3(a) are strikingly similar to those in Figure 3(b).

In order to identify the factors that derive the patterns in Figures 3(a) and 3(b), we utilize the findings of Körpeoğlu and Cho (2017). Specifically, using their equation (6), we can write $y^{*, N}$ as:

$$
y^{*, N}\left(a_{i}\right)=r\left(\frac{A}{c} G_{(1)}^{N-1}\left(a_{i}\right) E\left[\widetilde{a}_{(1)}^{N-1} \mid \widetilde{a}_{(1)}^{N-1}<a_{i}\right]\right) .
$$

In (19), there are two opposing forces that influence agent $i$ 's equilibrium output with an increase of $N$. First, a higher $N$ reduces agent $i$ 's probability of winning the contest, which corresponds to the probability of having a higher productivity than all other agents; i.e., $P\left(\widetilde{a}_{(1)}^{N-1}<a_{i}\right)=G_{(1)}^{N-1}\left(a_{i}\right)$, decreases with $N$. Second, Körpeoğlu and Cho (2017) show in their Proposition 1 that a larger $N$ raises the expected productivity of the runner-up, given that agent $i$ is the winner, $E\left[\widetilde{a}_{(1)}^{N-1} \mid \widetilde{a}_{(1)}^{N-1}<a_{i}\right]$. This second effect creates positive incentives for some agents to exert higher effort and improve output in order to win the contest. Depending on which of these two opposing forces dominates, agent $i$ may generate a better or worse output $y^{*, N}\left(a_{i}\right)$. Low-ability agents are hardly affected by increased competition because they already exert minimal effort due to low chances of wining. Moderate-ability agents tend to have lower effort and hence worse outputs because the impact of increased competition on their winning probability (i.e., $\left.G_{(1)}^{N}\left(a_{i}\right)\right)$ is dominant. High-ability superstars, who have higher winning probabilities, tend to increase effort and hence improve outputs because the incentives for exerting higher efforts to win the contest are stronger for them (i.e., an increase of $E\left[\widetilde{a}_{(1)}^{N-1} \mid \widetilde{a}_{(1)}^{N-1}<a_{i}\right]$ outweighs a decrease of $\left.G_{(1)}^{N}\left(a_{i}\right)\right)$. 


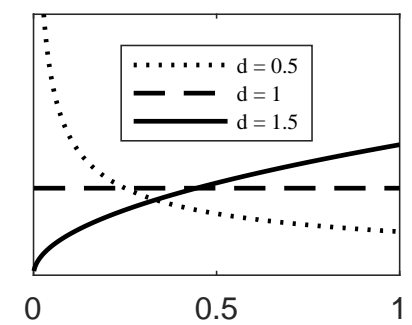

(a) $\tilde{a} \sim \operatorname{Beta}(\mathrm{d}, 1)$

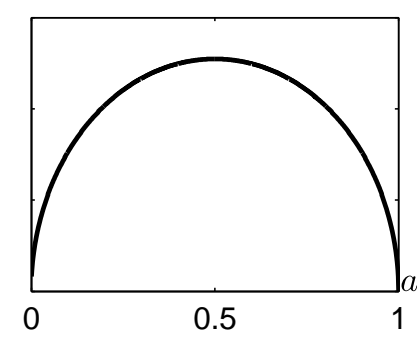

(b) $\tilde{a} \sim \operatorname{Beta}(1.5,1.5)$.

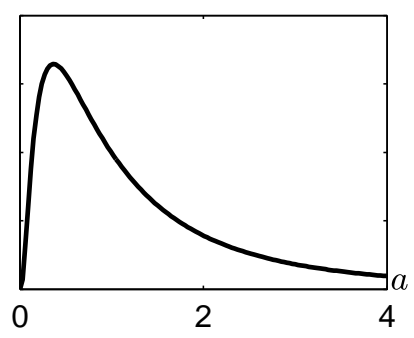

(c) $\tilde{a} \sim \log N(0,1)$.

Fig. 4 The density $g(a)$ for (a) beta distribution with parameters $d$ and 1, (b) beta distribution with parameters 1.5 and 1.5 , and (c) log-normal distribution with log-scale parameter 0 and shape parameter 1 .

Finally, we discuss when an open contest is optimal. An open contest is optimal when the organizer's profit increases with additional agents in the contest. Due to agents' heterogeneous response to additional agents in the contest, the organizer faces a trade-off when determining whether to allow more agents in the contest. More agents in the contest induce higher efforts from high-productivity agents, but reduce efforts of moderate-productivity agents. Because the organizer knows only the distribution of productivity levels of agents but does not know their exact productivity levels a priori (for example, it is possible that all agents have moderate productivity), it is not clear whether the organizer should hold an open contest. To illustrate when an open contest is optimal, we consider a special case with a CRRA effort function $r(e)=\theta \frac{e^{1-b}-1}{1-b}$ (with $b \in[0,1]$ ) and a generalized beta distribution that encompasses a beta distribution (when $\bar{a}=1$ ) with parameters $d$ and 1 including uniform as shown in Figure 4(a).

Proposition 6. When $r(e)=\theta \frac{e^{1-b}-1}{1-b}$, and $\widetilde{a} \in[0, \bar{a}]$ follows $G\left(a_{i}\right)=a_{i}^{d} / \bar{a}^{d}$ (where $b \in[0,1]$ and $d>0$ ), an open contest is optimal.

To build intuition for Proposition 6, we rewrite the organizer's profit $\Pi$ as follows:

$$
\Pi=\int_{\underline{a}}^{\bar{a}} y^{*, N}\left(a_{i}\right) g_{(1)}^{N}\left(a_{i}\right) d a_{i}-A=E\left[r\left(\widetilde{a}_{(1)}^{N} e^{*, N}\left(\widetilde{a}_{(1)}^{N}\right)\right)\right]-A .
$$

The number of agents $N$ has three effects on the organizer's profit $\Pi$. First, an increase in $N$ reduces the equilibrium effort $e^{*, N}$ of moderate-productivity agents (i.e., $e^{*, N}\left(a_{i}\right)$ is decreasing in $N$ for moderate values of $\left.a_{i}\right)$. Second, a higher $N$ raises $e^{*, N}$ for high-productivity agents. Third, the productivity level of the highest-productivity agent in the contest, $\widetilde{a}_{(1)}^{N}$, stochastically increases with $N$ (i.e., $\widetilde{a}_{(1)}^{N+1}$ first-order stochastically dominates $\left.\widetilde{a}_{(1)}^{N}\right)$. Proposition 6 indicates that the second and third effects outweigh the decreased effort from moderate-productivity agents. Thus, the organizer's profit increases with the number of agents $N$, so an open contest is optimal. While Proposition 6 shows the optimality of open contests for the generalized beta distribution, our supplementary numerical analysis verifies that this is also true for various other distributions such as symmetric beta distribution (Figure 4(b)) or a log-normal distribution (Figure 4(c)). 


\section{Conclusion and Future Research}

Innovation contests are becoming an ever more popular instrument for research and development. This transformation makes the research in the optimal design of contests of first-order importance. This chapter contributes to this research agenda by proposing a general model framework that encompasses commonly used models in the literature, and discussing two of the organizer's important decisions: How to award agents and whether to allow unrestricted entry to the contest. Our hope is that this chapter can serve as a building block for future contest research, and insights we provide can help both theorists and practitioners.

Research in innovation contests is still relatively young, and there are many interesting open questions. First, prior literature as well as this chapter has assumed a fixed contest duration, but the duration of a contest is also a strategic decision that organizers make in practice. The exploration of the optimal contest duration is an important future research direction. Second, we have adopted a relative compensation rule in awarding agents. Comparison of this compensation rule with other possible compensation rules may shed some light on why the relative compensation rule is so popular in practice. Third, we have considered a case in which the organizer is interested in a fixed number of solutions, and an interesting future research direction is to analyze a case in which the number of solutions organizer utilizes is endogenous to agents' solution qualities and the cost of implementing those solutions. Finally, characterizing equilibrium under both agent heterogeneity and uncertainty in a general form is an important research to pursue.

Overall, pioneering work in innovation contests has demonstrated that even the questions that have already been studied by prior economics literature can have completely different answers when considering the unique properties of innovation contests such as the impact of agents' uncertainty on the organizer's profit and the fact that the organizer is interested in only the best solution(s). Furthermore, the rapid growth of contest platforms such as InnoCentive poses new questions that were not relevant before. With abundant potential for interesting, practically relevant, and important research questions, innovation contests are an exciting area for future research.

\section{References}

Ales, L., S. Cho, E. Körpeoğlu. 2016. Innovation tournaments with multiple contributors. Working Paper, Carnegie Mellon University, Pittsburgh.

Ales, L., S. Cho., E. Körpeoğlu. 2017. Optimal award scheme in innovation tournaments. Operations Research 65(3) 693-702.

Bimpikis, K., S. Ehsani, M. Mostagir. 2016. Designing dynamic contests. Working Paper, Stanford University, Palo Alto.

Boudreau, K. J., C. E. Helfat, K. R. Lakhani, M. Menietti. 2012. Field evidence on individual behavior and performance in rank-order tournaments. Working Paper .

Che, Y. K., I. Gale. 2003. Optimal design of research contests. American Economic Review 93(3) 646-671. 
Dahan, E., H. Mendelson. 2001. An extreme-value model of concept testing. Management Science 47(1) 102 $-116$.

Erat, S., V. Krishnan. 2012. Managing delegated search over design spaces. Management Science 58(3) 606623.

Fullerton, R. L., R. P. McAfee. 1999. Auctioning entry into tournaments. Journal of Political Economy 107(3) 573-605.

Green, J. R., N. L. Stokey. 1983. A comparison of tournaments and contracts. Journal of Political Economy 91(3) 349-364.

Hu, M., L. Wang. 2017. Simultaneous vs. sequential crowdsourcing contests. Working paper, University of Toronto, Toronto.

Hvide, H. K. 2002. Tournament rewards and risk taking. Journal of Labor Economics 20(4) 877-898.

InnoCentive. 2017. Corporate info. http:/ /www.innocentive.com/about-us. Accessed on May 1, 2017.

James, H. 2014. Cultural representation in FIFA logos: 1990 - 2014. URL http:/ /www.logocontestreviews. com/cultural-representation-in-fifa-logos/.

Kalra, A., M. Shi. 2001. Designing optimal sales contests: A theoretical perspective. Marketing Science 20(2) 170-193.

Körpeoğlu, E., S. Cho. 2017. Incentives in contests with heterogeneous solvers. Management Science, Advance Online Publication. doi: 10.1287/mnsc.2017.2738.

Körpeoğlu, E., Ç . G. Körpeoğlu, İ. E. Hafalır. 2017. Contest among contest organizers. Working Paper, University College London, London.

Lazear, E. P., S. Rosen. 1981. Rank-order tournaments as optimum labor contracts. Journal of Political Economy 89(5) 841-864.

List, J. A., D. Van Soest, J. Stoop, H. Zhou. 2014. On the role of group size in tournaments: Theory and evidence from lab and field experiments. NBER Working Paper .

Liu, D., X. Geng, A. B. Whinston. 2007. Optimal design of consumer contests. Journal of Marketing 71(4) 140-155.

Mihm, J., J. Schlapp. 2016. Sourcing innovation: On feedback in contests. Working paper, INSEAD, Paris.

Moldovanu, B., A. Sela. 2001. The optimal allocation of prizes in contests. American Economic Review 91(3) 542-558.

Moldovanu, B., A. Sela. 2006. Contest architecture. Journal of Economic Theory 126 70-96.

Nalebuff, B. J., J. E. Stiglitz. 1983. Prizes and incentives: Towards a general theory of compensation and competition. Bell Journal of Economics 14(1) 21-43.

Nittala, L., V. Krishnan. 2016. Designing internal innovation contests. Working paper, University of California San Diego, San Diego. 
Shaked, M., J. G. Shanthikumar. 2007. Stochastic Orders. Springer, New York.

Siegel, R. 2009. All-pay contests. Econometrica 77(1) 71-92.

Stouras, K. I., J. Hutchison-Krupat, R. O. Chao. 2017. Motivating participation and effort in innovation contests. Working Paper University of Virginia, Charlottesville, VA.

Taylor, C. R. 1995. Digging for golden carrots: An analysis of research tournaments. American Economic Review 85(4) 872-890.

Terwiesch, C., Y. Xu. 2008. Innovation contests, open innovation, and multiagent problem solving. Management Science 54(9) 1529-1543.

TopCoder. 2017. Press releases. http://www.topcoder.com/press/. Accessed on May 19, 2017.

Tullock, G. 1967. The welfare costs of tariffs, monopolies, and theft. Economic Inquiry 5(3) 224-232.

Tullock, G. 1980. Efficient rent seeking. Tollison R. D. Buchanan, J. M., G. Tullock, eds., Toward a theory of the rent-seeking society. Texas A\&M University Press, TX.

Vojnović, M. 2015. Contest Theory. Cambridge University Press, New York, NY.

\section{Appendix}

Proof of Lemma 1(b). We use superscript $P$ to denote productivity-based model and superscript $C$ to denote cost-based model. Consider the productivity-based model with the output function $y\left(a_{i}, e_{i}, \widetilde{\xi}_{i}\right)=$ $r\left(a_{i} e_{i}\right)+\widetilde{\zeta}_{i}$, where $a_{i}$ is a heterogeneous productivity level. Let $v_{i}=a_{i} e_{i}$. Let $v^{*}\left(a_{i}\right)=a_{i} e^{*}\left(a_{i}\right)$ be a bestresponse function for agent $i$ with productivity $a_{i}$, where $e^{*}$ is the best-response effort. In this model, from agent $i$ 's perspective, another agent's output is a random variable $\widetilde{y}^{*, P} \equiv y^{*, P}\left(\widetilde{a}_{k}\right)=r\left(v^{*, P}\left(\widetilde{a}_{k}\right)\right)+\widetilde{\xi}_{k}$. Thus, in a productivity-based model, an agent $i$ solves:

$$
\max _{v_{i}} \sum_{j=1}^{N} P_{(j)}^{N}\left[v_{i}, v^{*, P}\right] A_{(j)}-\psi\left(v_{i} / a_{i}\right),
$$

where $P_{(j)}^{N}\left[v_{i}, v^{*, P}\right]=\int_{s \in \Xi} \frac{(N-1) !}{(j-1) !(N-j) !} P\left\{r\left(v_{i}\right)+s>\widetilde{y}^{*, P}\right\}^{N-j} P\left\{r\left(v_{i}\right)+s<\widetilde{y}^{*, P}\right\}^{j-1} h(s) d s$. In a cost-based model, all agents except agent $i$ have $v^{*, C}\left(c_{i}\right)$. We will construct a bijective mapping $\eta: R_{+} \rightarrow R_{+}$from an agent's $\operatorname{cost} c_{i}$ to a productivity $a_{i}$ (i.e., $\eta\left(c_{i}\right)=a_{i}$ ) such that given that all other agents have $v^{*, P}\left(a_{i}\right)=$ $v^{*, C}\left(c_{i}\right)$, agent $i$ will have the same best-response $v$. Define agent $i$ 's productivity as $a_{i}=\eta\left(c_{i}\right)=1 / c_{i}$. Given $v^{*, P}\left(a_{i}\right)=v^{*, C}\left(c_{i}\right)$, another agent's output is the following random variable: $\widetilde{y}^{*, C} \equiv r\left(v^{*, C}\left(\widetilde{c}_{j}\right)\right)+$ $\widetilde{\xi}_{j}=r\left(v^{*, P}\left(\widetilde{a}_{j}\right)\right)+\widetilde{\xi}_{j}=\widetilde{y}^{*, P}$. Then, in a cost-based model, $P_{(j)}^{N}\left[v_{i}, v^{*, C}\right]=\int_{s \in \Xi} \frac{(N-1) !}{(j-1) !(N-j) !} P\left\{r\left(v_{i}\right)+s>\right.$ $\left.\widetilde{y}^{*, C}\right\}^{N-j} P\left\{r\left(v_{i}\right)+s<\widetilde{y}^{*, C}\right\}^{j-1} h(s) d s$, and hence

$$
\arg \max _{v_{i}} \sum_{j=1}^{N} P_{(j)}^{N}\left[v_{i}, v^{*, C}\right] A_{(j)}-\psi\left(c_{i} v_{i}\right)=\arg \max _{v_{i}} \sum_{j=1}^{N} P_{(j)}^{N}\left[v_{i}, v^{*, P}\right] A_{(j)}-\psi\left(v_{i} / a_{i}\right),
$$

where the equality follows because $\widetilde{v}^{*, C}=\widetilde{v}^{*, P}$ and $c_{i}=\eta^{-1}\left(a_{i}\right)=1 / a_{i}$. Thus, the agent's problem in a costbased model is equivalent to the agent's problem in a productivity-based model. As a result, given that all 
other agents have output $v^{*, P}\left(a_{i}\right)=v^{*, C}\left(c_{i}\right)$, by using the mapping $\eta$, we obtain the same best response for agent $i$ under both models. Thus, in equilibrium, cost-based and productivity-based models satisfy $v^{*, P}\left(a_{i}\right)=v^{*, P}\left(\eta\left(c_{i}\right)\right)=v^{*, C}\left(c_{i}\right)=v^{*, C}\left(1 / a_{i}\right)$. Finally, using $\widetilde{a}=\eta(\widetilde{c})=1 / \widetilde{c}$, we obtain

$$
G\left(a_{i}\right)=P\left(\widetilde{a} \leq a_{i}\right)=P\left(1 / \widetilde{c} \leq a_{i}\right)=P\left(1 / a_{i} \leq \widetilde{c}\right)=1-\Phi\left(1 / a_{i}\right) .
$$

Lemma A1. In a productivity-based project with two prizes, a general productivity distribution $G$ and effort function $r$, an agent with productivity $a_{i}$ has the following equilibrium effort:

$$
e^{*}\left(a_{i}\right)=\frac{1}{a_{i}} \int_{\underline{a}}^{a_{i}} \frac{a}{c}\left[A_{(1)} g_{(1)}^{N-1}(a)+A_{(2)}(N-1)\left(g_{(1)}^{N-2}(a)-g_{(1)}^{N-1}(a)\right)\right] d a .
$$

Proof of Lemma A1. First, suppose that all agents except agent $i$ have output based on the best-response output function $y^{*}\left(a_{i}\right)$, which is assumed to be continuously differentiable and increasing in the productivity level $a_{i}$. We can write the best-response effort as $e^{*}\left(a_{i}\right)=r^{-1}\left(y^{*}\left(a_{i}\right)\right) / a_{i}$. Output $y_{i}$ of agent $i$ with productivity level $a_{i}$ is determined by the following problem:

$$
\max _{y_{i}} A_{(1)} G_{(1)}^{N-1}\left(\left(y^{*}\right)^{-1}\left(y_{i}\right)\right)+A_{(2)}(N-1)\left[G_{(1)}^{N-2}\left(\left(y^{*}\right)^{-1}\left(y_{i}\right)\right)-G_{(1)}^{N-1}\left(\left(y^{*}\right)^{-1}\left(y_{i}\right)\right)\right]-c r^{-1}\left(y_{i}\right) / a_{i} .
$$

The first-order condition evaluated at $y_{i}=y^{*}\left(a_{i}\right)$ gives (note that $y^{*}\left(a_{i}\right)=r\left(a_{i} e^{*}\left(a_{i}\right)\right)$ )

$$
\begin{aligned}
& {\left[A_{(1)} g_{(1)}^{N-1}\left(a_{i}\right)+A_{(2)}(N-1)\left(g_{(1)}^{N-2}\left(a_{i}\right)-g_{(1)}^{N-1}\left(a_{i}\right)\right)\right] \frac{1}{\left(y^{*}\right)^{\prime}\left(a_{i}\right)}-\frac{c}{a_{i} r^{\prime}\left(r^{-1}\left(y^{*}\left(a_{i}\right)\right)\right.} } \\
= & \frac{\left[A_{(1)} g_{(1)}^{N-1}\left(a_{i}\right)+A_{(2)}(N-1)\left(g_{(1)}^{N-2}\left(a_{i}\right)-g_{(1)}^{N-1}\left(a_{i}\right)\right)\right]}{r^{\prime}\left(a_{i} e^{*}\left(a_{i}\right)\right)\left[a_{i}\left(e^{*}\right)^{\prime}\left(a_{i}\right)+e^{*}\left(a_{i}\right)\right]}-\frac{c}{a_{i} r^{\prime}\left(a_{i} e^{*}\left(a_{i}\right)\right)}=0 .
\end{aligned}
$$

Multiplying both sides of (24) with $a_{i} r^{\prime}\left(a_{i} e^{*}\left(a_{i}\right)\right)\left[a_{i}\left(e^{*}\right)^{\prime}\left(a_{i}\right)+e^{*}\left(a_{i}\right)\right] / c$, we obtain

$$
\frac{a_{i}}{c}\left[A_{(1)} g_{(1)}^{N-1}\left(a_{i}\right)+A_{(2)}(N-1)\left(g_{(1)}^{N-2}\left(a_{i}\right)-g_{(1)}^{N-1}\left(a_{i}\right)\right)\right]-\left[a_{i}\left(e^{*}\right)^{\prime}\left(a_{i}\right)+e^{*}\left(a_{i}\right)\right]=0 .
$$

Since $y^{*}\left(a_{i}\right)$ is increasing, in a contest with $N>2$, the least productive agent cannot win $A_{(1)}$ or $A_{(2)}$, so exerts zero effort (i.e., $\left.e^{*}(\underline{a})=0\right)$. Thus, $e^{*}\left(a_{i}\right)=\frac{1}{a_{i}} \int_{\underline{a}}^{a_{i}} \frac{a}{c}\left[A_{(1)} g_{(1)}^{N-1}(a)+A_{(2)}(N-1)\left(g_{(1)}^{N-2}(a)-g_{(1)}^{N-1}(a)\right)\right] d a$ is the solution to the solution of (25). Therefore, the equilibrium output function $y^{*}\left(a_{i}\right)$ is

$$
y^{*}\left(a_{i}\right)=r\left(\int_{\underline{a}}^{a_{i}} \frac{a}{c}\left[A_{(1)} g_{(1)}^{N-1}(a)+A_{(2)}(N-1)\left(g_{(1)}^{N-2}(a)-g_{(1)}^{N-1}(a)\right)\right] d a\right) .
$$

Finally, we verify that the equilibrium output function $y^{*}\left(a_{i}\right)$ is continuously differentiable and increasing in $a_{i}$. Since all of the terms inside the integral in (26) are continuously differentiable in $a_{i}$, and $r$ is continuously differentiable, so is $y^{*}$. Taking the derivative of $y^{*}\left(a_{i}\right)$ with respect to $a_{i}$, we obtain $\left(y^{*}\right)^{\prime}\left(a_{i}\right)=r^{\prime}\left(\int_{\underline{a}}^{a_{i}} \phi(a) d a\right) \times \phi\left(a_{i}\right)$, where $\phi\left(a_{i}\right) \equiv \frac{a_{i}}{c}\left[A_{(1)} g_{(1)}^{N-1}\left(a_{i}\right)+A_{(2)}(N-1)\left(g_{(1)}^{N-2}\left(a_{i}\right)-g_{(1)}^{N-1}\left(a_{i}\right)\right)\right]$. Thus, $y^{*}$ is increasing because $r^{\prime}>0$, and $A_{(1)} \geq A_{(2)}$ implies

$$
\begin{aligned}
\phi\left(a_{i}\right) & \geq \frac{a_{i}}{c}\left[A_{(2)} g_{(1)}^{N-1}\left(a_{i}\right)+A_{(2)}(N-1)\left(g_{(1)}^{N-2}\left(a_{i}\right)-g_{(1)}^{N-1}\left(a_{i}\right)\right)\right] \\
& =\frac{a_{i} A_{(2)}}{c}(N-2)(N-1) G\left(a_{i}\right)^{N-3} g\left(a_{i}\right)\left[1-G\left(a_{i}\right)\right]>0 .
\end{aligned}
$$


Then, $y^{*}\left(a_{i}\right)=r\left(a_{i} e^{*}\left(a_{i}\right)\right)$ is the agent's equilibrium output proposed in the lemma.

Proof of Proposition 5. The derivative of $V$ with respect to $\alpha$ is

$$
\frac{\partial V}{\partial \alpha}=\int_{\underline{a}}^{\bar{a}} a_{i} r^{\prime}\left(a_{i} e^{*}\left(a_{i}\right)\right) \frac{\partial e^{*}\left(a_{i}\right)}{\partial \alpha} g_{(1)}^{N}\left(a_{i}\right) d a_{i} .
$$

To evaluate (27), we need the equilibrium effort $e^{*}\left(a_{i}\right)$ and its derivative with respect to $\alpha$. If we substitute $A_{(1)}=(1-\alpha) A, A_{(2)}=\alpha A$ into (23), and take derivative of $e^{*}\left(a_{i}\right)$ with respect to $\alpha$, we obtain $\frac{\partial e^{*}\left(a_{i}\right)}{\partial \alpha}=$ $\frac{A}{a_{i}} \int_{\underline{a}}^{a_{i}} \frac{a}{c}\left[(N-1) g_{(1)}^{N-2}(a)-N g_{(1)}^{N-1}(a)\right] d a$. Under CRRA function, noting that $r^{\prime}(e)=\theta e^{-b},(27)$ becomes

$$
\frac{\partial V}{\partial \alpha}=\theta \int_{\underline{a}}^{\bar{a}} a_{i}\left(a_{i} e^{*}\left(a_{i}\right)\right)^{-b} \frac{\partial e^{*}\left(a_{i}\right)}{\partial \alpha} g_{(1)}^{N}\left(a_{i}\right) d a_{i} .
$$

If $\frac{\partial V}{\partial \alpha}>0$, it is optimal for the organizer to set $\alpha>0$ which proves the second part of the proposition.

When $b=0$ (i.e., $r(e)=\theta e$ ), (28) becomes

$$
\begin{aligned}
\frac{\partial V}{\partial \alpha} & =\int_{\underline{a}}^{\bar{a}} \int_{\underline{a}}^{a_{i}} \frac{A \theta a}{c}\left[(N-1) g_{(1)}^{N-2}(a)-N g_{(1)}^{N-1}(a)\right] g_{(1)}^{N}\left(a_{i}\right) d a d a_{i} \\
& =\int_{\underline{a}}^{\bar{a}} \frac{A \theta a}{c}\left[g_{(2)}^{N-1}(a)-g_{(2)}^{N}(a)\right] \frac{\left(1-G_{(1)}^{N}(a)\right)}{1-G(a)} d a,
\end{aligned}
$$

because $N g_{(1)}^{N-1}(a)=N(N-1) G(a)^{N-2} g(a)=\frac{N(N-1)(1-G(a)) G(a)^{N-2} g(a)}{(1-G(a))}=\frac{g_{(2)}^{N}(a)}{(1-G(a))}$. Thus,

$$
\left.\frac{\partial V}{\partial \alpha}=\frac{A \theta}{c}\left(E\left[\widetilde{a}_{(2)}^{N-1}\left(\sum_{j=1}^{N-1} G\left(\widetilde{a}_{(2)}^{N-1}\right)^{j}\right)\right]-E\left[\widetilde{a}_{(2)}^{N}\left(\sum_{j=1}^{N-1} G\left(\widetilde{a}_{(2)}^{N}\right)^{j}\right)^{N}\right)\right]\right)<0,
$$

where the inequality follows because $a\left(\sum_{j=0}^{N-1} G(a)^{j}\right)$ is an increasing function of $a$, and $\widetilde{a}_{(2)}^{N}$ is larger than $\widetilde{a}_{(2)}^{N-1}$ in the sense of first-order stochastic dominance (cf. Theorem 1.A.8 of Shaked and Shanthikumar 2007). Thus, it is optimal for the organizer to set $\alpha=0$. When $b>0$, it is not difficult to verify that $\frac{\partial V}{\partial \alpha}$ is continuous in $b$ because all terms in (28) are continuous in $b$. Then, for sufficiently small $b$, we have $\frac{\partial V}{\partial \alpha}<0$. Therefore, there exists $b_{0}>0$ such that for all $b<b_{0}$, it is optimal for the organizer to set $A_{(1)}=A$ and $A_{(2)}=0$.

Proof of Proposition 6. Suppose that $\widetilde{a} \in[0, \bar{a}]$ follows $G\left(a_{i}\right)=a_{i}^{d} / \bar{a}^{d}$. Substituting the effort $e^{*}\left(a_{i}\right)=$ $\frac{A d(N-1)}{c(d(N-1)+1)}\left(\frac{a_{i}}{\bar{a}}\right)^{d(N-1)}$ and $r(e)=\theta \frac{e^{1-b}-1}{1-b}$ in $y^{*}\left(a_{i}\right)=r\left(a_{i} e^{*}\left(a_{i}\right)\right)$ yields

$$
y^{*}\left(a_{i}\right)=\frac{\theta}{1-b}\left(\frac{a_{i} A d(N-1)}{c(d(N-1)+1)}\left(\frac{a_{i}}{\bar{a}}\right)^{d(N-1)}\right)^{1-b}-\frac{1}{1-b} .
$$

Noting that $g_{(1)}^{N}\left(a_{i}\right)=n G\left(a_{i}\right)^{N-1} g\left(a_{i}\right)=\frac{N d}{\bar{a}}\left(\frac{a_{i}}{\bar{a}}\right)^{d(N-1)+d-1}$, we can the organizer's profit as

$$
\begin{aligned}
\Pi & =\int_{0}^{\bar{a}}\left[\frac{\bar{a} A d(N-1)}{c(d(N-1)+1)}\right]^{1-b} \frac{\left(\frac{a_{i}}{\bar{a}}\right)^{[d(N-1)+1](1-b)}-1}{1-b}\left[\frac{N d}{\bar{a}}\left(\frac{a_{i}}{\bar{a}}\right)^{d(N-1)+d-1}\right] d a_{i}-A \\
& =\left[\frac{\bar{a} A d(N-1)}{c(d(N-1)+1)}\right]^{1-b} \frac{d}{(1-b)}\left[\frac{N}{[d(N-1)+1](1-b)+N d}\right]-\frac{1}{1-b}-A .
\end{aligned}
$$


Let $W(N)=\frac{\bar{a} d(N-1)}{c(d(N-1)+1)}\left(\left[\frac{d N}{[d(N-1)+1](1-b)+N d}\right]\right)^{\frac{1}{1-b}}$. Noting that $\Pi=\frac{A^{1-b} W(N)^{1-b}}{1-b}-\frac{1}{1-b}-A$ is concave in $A$, the optimal winner prize $A^{*}=W(N)^{\frac{1-b}{b}}$. Substituting $A^{*}$ back to $\Pi$, we get

$$
\Pi=\frac{W(N)^{(1-b)\left(\frac{1-b}{b}+1\right)}}{1-b}-\frac{1}{1-b}-W(N)^{\frac{1-b}{b}}=W(N)^{\frac{1-b}{b}}\left[\frac{1}{1-b}-1\right]-\frac{1}{1-b}=\frac{W(N)^{\frac{1-b}{b}}}{1-b} b-\frac{1}{1-b} .
$$

Because $W(N)$ is increasing with $N$, so is $\Pi$. 